\title{
Does Low-Dose Intravenous Methylprednisolone Pulse Therapy Produce Unacceptable Adverse Effects in Children?*
}

\author{
Daishi Hirano $^{1,2}$, Shuichiro Fujinaga ${ }^{1}$, Amane Endo ${ }^{1}$, Tsuneki Watanabe ${ }^{1}$, Hiroyuki Ida ${ }^{2}$ \\ ${ }^{1}$ Division of Nephrology, Saitama Children's Medical Center, Saitama, Japan \\ ${ }^{2}$ Department of Pediatrics, Jikei University School of Medicine, Tokyo, Japan \\ Email: bqx1976@hotmail.com
}

Received September 22, 2013; revised October 20, 2013; accepted November 15, 2013

Copyright (C) 2013 Daishi Hirano et al. This is an open access article distributed under the Creative Commons Attribution License, which permits unrestricted use, distribution, and reproduction in any medium, provided the original work is properly cited.

\begin{abstract}
Background: Intravenous methylprednisolone pulse therapy has been used since the late 1960s for acute transplant rejection or severe renal involvement in systemic lupus erythematosus and primary glomerulonephritis. However, reports of serious adverse effects such as life-threatening cardiac arrhythmias and sudden death raise questions about its safety. Objective: To investigate the incidence of significant adverse effects associated with low-dose methylprednisolone pulse therapy (LDMPT) in pediatric patients. Methods: We retrospectively analyzed adverse effects during and after LDMPT in 68 patients (median age: 11.4 years; $43 \%$ male) with various glomerular diseases who were admitted to Saitama Children's Medical Center between April 2007 and December 2010. LDMPT consisted of pulse methylprednisolone (15 - $20 \mathrm{mg} / \mathrm{kg}$; maximum $600 \mathrm{mg} / \mathrm{d})$ for 3 consecutive days weekly for 2 - 3 weeks. Results: Although adverse effects occurred in 54 of 68 patients (79\%), most were mild and transient. Transient glycosuria was noted in 46 patients $(68 \%)$, hypertension in $6(9 \%)$, elevated intraocular pressure in $6(9 \%)$, hypokalemia in $5(7 \%)$, and liver damage in $2(3 \%)$. No late-onset adverse effects such as osteoporotic fractures, steroid diabetes mellitus, or short stature were observed. Conclusion: LDMPT appears to be relatively safe and well tolerated in children with various glomerular diseases.
\end{abstract}

Keywords: Methylprednisolone Pulse Therapy; Adverse Effects; Side Effects; Steroid; Children

\section{Introduction}

Conventional high-dose intravenous methylprednisolone pulse therapy (HDMPT) first came into clinical use over 40 years ago to treat acute renal graft rejection. Although MPT has since become an essential tool for clinicians treating various kidney diseases, little is known about its mechanism of effect and magnitude of associated adverse effects [1-5]. The most serious problems associated with HDMPT in adults are cardiovascular reactions, including death $[6,7]$, but in children, the adverse effects are reported by some researchers to be quite different [8]. The most common adverse effects in children are psychiatric reactions (e.g., mood alteration, hyperactivity, psychosis, disorientation, and sleep disturbances), which are seen in about $10 \%$ of children receiving HDMPT [8]. According to recent reports, these adverse effects are associated

\footnotetext{
${ }^{*}$ The authors have no conflicts of interest to declare.
}

with higher doses of methylprednisolone (mPSL) and higher rates of intravenous administration [5,9]. In fact, Klein-Gitelman et al. reported that remarkable improvement was observed in some children with a decreased dose or an increased time interval between administrations [8]. However, adverse effects of decreased doses of mPSL have not been clearly delineated. Based on these reports, we now treat a variety of kidney diseases using "low-dose" MPT (LDMPT), which extends the infusion time $(>2 \mathrm{~h})$ and reduces the dosage, in order to reduce adverse effects. The objective of this study was to determine the frequency and severity of adverse effects associated with LDMPT in pediatric patients.

\section{Patients and Materials}

\subsection{Patients}

In this retrospective case series study, we collected and 
analyzed data on 196 courses of LDMPT given to 68 children (29 boys, 39 girls) at the Saitama Children's Medical Center in Saitama, Japan from April 2007 through December 2010. Informed consent was obtained from the patients' parents. Prior to LDMPT, all patients were given a complete physical examination in order to screen and exclude those with known infections, uncontrolled hypertension, congestive heart failure, or uncontrolled diabetes mellitus. The physical examination included measurement and evaluation of systolic blood pressure, diastolic blood pressure, and baseline chemistry parameters. Only data from patients with normal values for these parameters were included in the analysis.

\subsection{Low-Dose Methylprednisolone Pulse Therapy}

LDMPT was defined as mPSL administered at a dose of $15-20 \mathrm{mg} / \mathrm{kg} / \mathrm{d}$ (maximum: $600 \mathrm{mg} / \mathrm{d}$ ) in $50-100 \mathrm{ml}$ of $5 \%$ dextrose solution given intravenously over $2 \mathrm{~h}$ for 3 consecutive days weekly for 2 - 3 weeks. In comparison, the "high dose" for HDMPT is $30 \mathrm{mg} / \mathrm{kg} / \mathrm{d}$ (maximum 1 $\mathrm{g} / \mathrm{d}$ ). All LDMPT administrations took place in a hospital setting under physician supervision, with monitoring of vital signs every $30 \mathrm{~min}$ or more frequently if an abnormality was found or the patient experienced any symptoms.

\subsection{Adverse Effects}

Adverse effects were defined as any new symptom or sign (e.g., skin rash, headache, dizziness, dysgeusia, chest pain, palpitations, dyspnea, nausea, osteonecrosis of the femoral head, psychiatric reaction, steroid diabetes mellitus, and short stature) that appeared during or after each LDMPT course Adverse effects were categorized as either early or late onset. Early-onset adverse effects were defined as those appearing during LDMPT or within 4 weeks after initiation of LDMPT, and late-onset adverse effects were defined as those appearing $\geq 4$ weeks after initiation of LDMPT. For late-onset adverse effects such as osteonecrosis of the femoral head or short stature, we analyzed data from 34 children with IgA nephropathy (IgAN) who had been followed for $\geq 1$ year. We identified other adverse sequelae from diagnostic procedures such as laboratory tests or from records of vital signs (blood pressure and heart rate changes) outside the normal range. We retrieved data from medical records relevant to the number, frequency, and total dosage of LDMPT administrations. We also collected information pertaining to diagnosis, age, sex, laboratory data at the time of reaction, other medications received, and history of other adverse drug events.

\subsection{Statistical Analysis}

Continuous variables were expressed as median and range; categorical variables were expressed as number and percentage values. Statistical analysis was performed using SAS software, version 9.3 (SAS Institute, Cary, NC). Statistical significance was defined as $p<0.05$.

\section{Results}

\subsection{Patient Characteristics}

Table 1 summarizes the characteristics of patients treated with LDMPT. The patients were 29 boys (43\%) and 39 girls $(57 \%)$, with a median age was 11.4 years (range: 1.4 - 18.1 years), who together had received 196 LDMPT treatments. Underlying disease was $\operatorname{IgAN}$ in 35 patients (51\%), Henoch-Schönlein purpura nephritis in $20(29 \%)$, membranoproliferative glomerulonephritis in $6(9 \%)$, nephrotic syndrome in $5(7 \%)$, and other in 2 (4\%). Sixty-six children received $<3$ LDMPT treatments and 2 received $\geq 4$ LDMPT treatments. Median mPSL dose was $15.0 \mathrm{mg} / \mathrm{kg} / \mathrm{d}$ (range: 7.9 - $24.1 \mathrm{mg} / \mathrm{kg} / \mathrm{d}$ ); since the maximum mPSL dosage that could be given was $600 \mathrm{mg} / \mathrm{d}$, the dose could be $<15 \mathrm{mg} / \mathrm{kg}$, but could be slightly $>20$ $\mathrm{mg} / \mathrm{kg}$ because standard size ampules $(100 \mathrm{mg}$ or $250 \mathrm{mg}$ ) were used.

\subsection{Early-Onset Adverse Effects}

Early-onset adverse effects occurred in 54 of 68 children (79\%), but most were mild, transient, and required no medical treatment (Table 2). Transient glycosuria, the most common symptom, was seen in 46 children (68\%). It occurred within the first 3 doses of LDMPT, but usually disappeared soon after LDMPT course ended. Mild hypertension was noted in 6 children $(9 \%)$, but all required antihypertensive medication to control blood pressure. These children continued LDMPT with the antihypertensive agents without further significant ad-

Table 1. Baseline characteristics of patients receiving lowdose methylprednisolone pulse therapy (LDMPT).

\begin{tabular}{cc}
\hline Total number of patients & 68 \\
\hline Sex (male/female) & $29 / 39$ \\
Median age, years (range) & $11.4(1.4-18.1)$ \\
Number of LDMPT courses & 196 \\
Total number of LDMPT courses & $181 / 66$ \\
1 - 3 times/patient & $15 / 2$ \\
$>3$ times/patient & \\
Disease, n (\%) & $35(51)$ \\
IgA nephropathy & $20(29)$ \\
Henoch-Schönlein purpura nephritis & $6(9)$ \\
Membranoproliferative glomerulonephritis & $5(7)$ \\
Nephrotic syndrome & $2(4)$ \\
\hline
\end{tabular}


Table 2. Adverse effects during and after low-dose methylprednisolone pulse therapy.

\begin{tabular}{cc}
\hline Adverse effect & $\mathbf{n}(\%)$ \\
\hline Transient glycosuria & $46(68)$ \\
Hypertension & $6(9)$ \\
Elevated intraocular pressure & $6(9)$ \\
Hypokalemia & $5(7)$ \\
Elevated liver transaminase levels & $2(3)$ \\
Severe adverse effect & \\
Bacterial infection & $0(0)$ \\
Cardiac arrhythmia & $0(0)$ \\
Bradycardia & $0(0)$ \\
Thrombosis & $0(0)$ \\
Peptic ulcer & $0(0)$ \\
Acute pancreatitis & $0(0)$ \\
Neuropsychiatric disorder & $0(0)$ \\
\hline
\end{tabular}

verse effects. Other adverse effects associated with LDMPT included elevated intraocular pressure, hypokalemia, and liver damage, which were detected in 6 $(9 \%), 5(7 \%)$, and $2(3 \%)$ patients, respectively. None of the children needed treatment for severe adverse effects such as cardiac arrhythmia, bradycardia, bacterial infection, thrombosis, peptic ulcer, acute pancreatitis, or neuropsychiatric disorder.

\subsection{Late-Onset Adverse Effects}

To assess late-onset adverse effects, we analyzed data from the 34 children with IgAN who had been given bone density tests while being followed for $\geq 1$ year. Median height of these patients at last observation was -0.09 standard deviation score (SDS) for normal-for-age height (range: -1.57 to +2.28 SDS), and none of these patients had abnormally short stature ( -2.0 SDS). There were no statistical differences between the median SDS before LDMPT and that for the last observation. We found no documented case of osteonecrosis.

\section{Discussion}

This study investigated the frequency and severity of adverse effects associated with LDMPT in pediatric patients. Although MPT has become an important therapeutic modality for clinicians treating autoimmune diseases, there is as yet insufficient evidence for its mechanism of action and magnitude of benefits and adverse effects [10]. Moreover, its adverse effects are reported to differ between adults and children.

In a previous prospective study of the frequency and severity of adverse reactions associated with HDMPT in children with rheumatic disease, Klein-Gitelman et al. found that $46 \%(22 / 213)$ had adverse reactions, although none required hospitalization [8]. Baethge et al. also reported that HDMPT had an acceptably low incidence of significant adverse effects in adults [11]. In contrast, Garrett et al. found adverse effects in 56\% of a group of adult patients with rheumatic disease who received HDMPT, of whom $24 \%$ needed medical intervention [12]. Undoubtedly MPT can lead to severe life-threatening complications such as cardiac arrhythmias, sudden death, circulatory collapse, and cardiac arrest [6,7]. However, most of the reported cases have involved adults with underlying cardiac disease, usually following rapid administration of large doses of mPSL ( $>500 \mathrm{mg}$ administered over $<10 \mathrm{~min}$ ) [6]. In human studies, pulse dosing of intravenous mPSL alters the stimulation threshold of myocardial cells. It also alters serum potassium and urinary excretion of both potassium and sodium. These changes might conceivably alter electrolyte shifts across the myocardial cell membrane $[13,14]$. In our study, patients were free of underlying cardiac disease prior to the onset of renal disease or intractable hypertension. Also, our patients were given mPSL over $2 \mathrm{~h}$, with frequent monitoring of vital signs, and no episodes of serious cardiovascular adverse effects occurred. However, some did experience hypertension that responded rapidly to appropriate intervention.

The most common adverse effect among our pediatric patients was renal glycosuria. Glucocorticoids impair glucose metabolism mainly by inducing insulin resistance and increasing hepatic gluconeogenesis. Insulin resistance appears to occur at both receptor and post-receptor sites, and variations between glucocorticoids with regard to insulin binding do exist. Glucocorticoids can also induce hyperglycemia through the stimulation of $\alpha$-cells, leading to hyperglucagonemia and increased glycogenolysis $[15,16]$. These hyperglycemic effects are responsible for the so-called steroid diabetes observed in $25 \%$ (6) of subjects treated with long-term oral corticosteroid therapy $[17,18]$. Unlike oral treatment, however, there is insufficient quantitative data on the hyperglycemic effects of intravenous MPT. What we do know is that in a study by Baethge et al. 19\% (16/84) of adults with rheumatic disease had hyperglycemic effects associated with HDMPT, with only 1 insulin-dependent patient requiring hypoglycemic therapeutic adjustment [11]. Feldman-Billard et al.'s analysis of serial fasting blood glucose measurements in 198 nondiabetic and 28 diabetic patients with eye disease treated by MPT revealed that the diabetic patients tended to show a cumulative hyperglycemic effect of iterative MPT, whereas nondiabetic patients were able to spontaneously regulate MPT-induced hyperglycemia [19]. These findings indicate that close glycemic monitoring is not needed during MPT with nondiabetic patients. 
In a cohort of 539 patients with systemic lupus erythematosus, Zonana-Nacach et al. found no association between intravenous steroid therapy and osteoporotic fractures, but did find an association with high dose oral steroids, reporting a strong association between cumulative prednisolone dose and adverse effects [20]. In our study, we fortunately did not experience late-onset adverse effects such as osteoporotic fractures, steroid diabetes mellitus, and short stature. This might be because we administered a lower dose of oral mPSL $(1 \mathrm{mg} / \mathrm{kg} / \mathrm{d}$, maximum $30 \mathrm{mg} / \mathrm{d}$ ) on alternate days after LDMPT.

The minimum effective dose of mPSL is unclear. While one study has suggested that doses as low as 320 mg administered intravenously or intramuscularly are as effective as $1 \mathrm{~g}$ of intravenous mPSL [21], another study has shown that reducing the intravenous mPSL dose from $1 \mathrm{~g}$ to $500 \mathrm{mg}$ results in a substantial loss of efficacy [22]. Our findings suggest that LDMPT could be a potentially effective and safe alternative for pediatric patients with various glomerular diseases. However, more studies are needed to further characterize the optimal dosages and mechanisms of action of MPT.

\section{Limitations}

The present study was a retrospective analysis of treatment performed at a single center, so there may be some selection bias in the cohort. The single center cohort design also limits the external validity of our findings. In addition, we could not determine the true frequency of minor adverse effects such as metallic taste and facial flushing because of the manner in which this retrospective study was performed. However, we are confident that we did not miss any serious treatment complications.

\section{Conclusion}

This case series indicated that LDMPT might have an acceptably low risk of significant adverse effects and therefore can be an effective treatment modality for pediatric patients with various glomerular diseases.

\section{Tables}

Table 1 summarizes the characteristics of patients treated with LDMPT.

Adverse effects were categorized as either early or late onset. And Table 2 summarizes the adverse effects during and after low-dose methylprednisolone pulse therapy.

\section{REFERENCES}

[1] E. S. Cathcart, B. A. Idelson, M. A. Scheinberg and W. G. Couser, "Beneficial Effects of Methylprednisolone 'Pulse' Therapy in Diffuse Proliferative Lupus Nephritis," The Lancet, Vol. 307, No. 7952, 1976, pp. 163-166.

\section{http://dx.doi.org/10.1016/S0140-6736(76)91272-1}

[2] R. J. Levinsky, J. S. Cameron and J. F. Soothill, "Serum Immune Complexes and Disease Activity in Lupus Nephritis," The Lancet, Vol. 309, No. 8011, 1977, pp. 564567. http://dx.doi.org/10.1016/S0140-6736(77)91998-5

[3] A. Sinha and A. Bagg, "Pulse Steroid Therapy," The Indian Journal of Pediatrics, Vol. 75, No. 10, 2008, pp. 1057-1066. http://dx.doi.org/10.1007/s12098-008-0210-7

[4] M. Ohya, H. Otani, Y. Minami, S. Yamanaka, T. Mima, S. Negi, S. Yukawa and T. Shigematsu, "Tonsillectomy with Steroid Pulse Therapy Has More Effect on the Relapse Rate than Steroid Pulse Monotherapy in IgA Nephropathy Patients," Clinical Nephrology, Vol. 80, No. 1, 2013, pp. 47-52. http://dx.doi.org/10.5414/CN107861

[5] D. Gracey, R. Garsia, W. Britton and P. McKenzie. "Rapid Recovery of Renal Function after Pulse Steroid Therapy in a Human Immunodeficiency Virus-Infected Patient with Glomerulonephritis," Internal Medicine Journal, Vol. 42, No. 12, 2012, pp. 1363-1365.

http://dx.doi.org/10.1111/imj.12014

[6] B. L. Erstad, "Severe Cardiovascular Adverse Effects in Association with Acute, High-Dose Corticosteroid Administration," Drug Intelligence and Clinical Pharmacy, Vol. 23, No. 12, 1989, pp. 1019-1023.

[7] B. A. McDougal, F. C. Whittier and D. E. Cross, "Sudden Death after Bolus Steroid Therapy for Acute Rejection," Transplantation Proceedings, Vol. 8, No. 3, 1976, pp. 493-496.

[8] M. S. Klein-Gitelman and L. M. Pachman, "Intravenous Corticosteroids: Adverse Reactions Are More Variable than Expected in Children," The Journal of Rheumatology, Vol. 25, No. 10, 1998, pp. 1995-2002.

[9] "The Boston Collaborative Drug Surveillance Program. Acute Adverse Reactions to Prednisone in Relation to Dosage," Clinical Pharmacology \& Therapeutics, Vol. 13, No. 5, 1972, pp. 694-698.

[10] M. D. Smith, M. J. Ahern and P. J. Roberts-Thomson, "Pulse Methylprednisolone Therapy in Rheumatoid Arthritis: Unproved Therapy, Unjustified Therapy, or Effective Adjunctive Treatment?" Annals of the Rheumatic Diseases, Vol. 49, No. 4, 1990, pp. 265-267. http://dx.doi.org/10.1136/ard.49.4.265

[11] B. A. Baethge, M. D. Lidsky and J. W. Goldber, "A Study of Adverse Effects of High-Dose Intravenous (Pulse) Methylprednisolone Therapy in Patients with Rheumatic Disease," Annals of Pharmacotherapy, Vol. 25, No. 3, 1992, pp. 316-320.

[12] R. Garrett and H. Paulus, "Complications of Intravenous Methylprednisolone Pulse Therapy (Abstract)," Arthritis \& Rheumatism, Vol. 23, No. 6, 1980, p. 677.

[13] S. Fujimoto, H. Kondoh, Y. Yamamoto, S. Hisanaga and K. Tanaka, "Holter Electrocardiogram Monitoring in Nephrotic Patients during Methylprednisolone Pulse Therapy," American Journal of Nephrology, Vol. 10, No. 3, 1990, pp. 231-236. http://dx.doi.org/10.1159/000168087

[14] C. Svorcík and L. Bicíková, "Effect of Drugs on the Stimulation Threshold of the Human Heart," Cor et Vasa, Vol. 20, No. 3, 1978, pp. 184-195. 
[15] G. Pagano, P. Cavallo-Perin, M. Cassader, A. Bruno, A. Ozzello, P. Masciola, A. M. Dall'omo and B. Imbimbo, "An in Vivo and in Vitro Study of the Mechanism of Prednisone-Induced Insulin Resistance in Healthy Subjects," The Journal of Clinical Investigation, Vol. 72, No. 5, 1983, pp. 1814-1820. http://dx.doi.org/10.1172/JCI111141

[16] M. McMahon, J. Gerich and R. Rizza, "Effects of Glucocorticoids on Carbohydrate Metabolism," Diabetes/Metabolism Reviews, Vol. 4, No. 1, 1988, pp. 17-30. http://dx.doi.org/10.1002/dmr.5610040105

[17] M. K. Pandit, J. Burke, A. B. Gustafson, A. Minocha and A. N. Peiris, "Drug-Induced Disorders of Glucose Tolerance," Annals of Internal Medicine, Vol. 118, No. 7, 1993, pp. 529-539.

http://dx.doi.org/10.7326/0003-4819-118-7-199304010-0 $\underline{0008}$

[18] J. H. Gurwitz, R. L. Bohn, R. J. Glynn, M. Monane, H. Mogun and J. Avorn, "Glucocorticoids and the Risk for Initiation of Hypoglycemic Therap," Archives of Internal Medicine, Vol. 154, No. 1, 1994, pp. 97-101. http://dx.doi.org/10.1001/archinte.1994.00420010131015
[19] S. Feldman-Billard, B. Lissak, R. Benrabah, R. Kassaei and E. Héron, "Intravenous Pulse Methylprednisolone Therapy in Eye Disease," Ophthalmology, Vol. 110, No. 12, 2003, pp. 2369-2371. http://dx.doi.org/10.1016/S0161-6420(03)00818-2

[20] A. Zonana-Nacach, S. G. Barr, L. S. Magder and M. Petri, "Damage in Systemic Lupus Erythematosus and Its Association with Corticosteroids," Arthritis \& Rheumatism, Vol. 43, No. 8, 2000, pp. 1801-1808. http://dx.doi.org/10.1002/1529-0131(200008)43:8<1801:: AID-ANR16>3.0.CO;2-O

[21] M. Radia and D. E. Furst, "Comparison of Three Pulse Methylprednisolone Regimens in the Treatment of Rheumatoid Arthritis," The Journal of Rheumatology, Vol. 15, No. 2, 1998, pp. 242-246.

[22] M. E. Shipley, P. A. Bacon, H. Berry, B. L. Hazleman, R. D. Sturrock, D. R. Swinson and I. A. Williams, "Pulsed Methylprednisolone in Active Early Rheumatoid Disease: A Dose-Ranging Study," British Journal of Rheumatology, Vol. 27, No. 3, 1988, pp. 211-214. http://dx.doi.org/10.1093/rheumatology/27.3.211 